\title{
Interval non-edge-colorable bipartite graphs and multigraphs
}

\author{
Petros A. Petrosyan ${ }^{\text {ab* }}$, Hrant H. Khachatrian ${ }^{\text {a }}$ \\ ${ }^{a}$ Department of Informatics and Applied Mathematics, \\ Yerevan State University, 0025, Armenia \\ ${ }^{\mathrm{b}}$ Institute for Informatics and Automation Problems, \\ National Academy of Sciences, 0014, Armenia
}

An edge-coloring of a graph $G$ with colors $1, \ldots, t$ is called an interval $t$-coloring if all colors are used, and the colors of edges incident to any vertex of $G$ are distinct and form an interval of integers. In 1991 Erdős constructed a bipartite graph with 27 vertices and maximum degree 13 which has no interval coloring. Erdös's counterexample is the smallest (in a sense of maximum degree) known bipartite graph which is not interval colorable. On the other hand, in 1992 Hansen showed that all bipartite graphs with maximum degree at most 3 have an interval coloring. In this paper we give some methods for constructing of interval non-edge-colorable bipartite graphs. In particular, by these methods, we construct three bipartite graphs which have no interval coloring, contain 20,19,21 vertices and have maximum degree $11,12,13$, respectively. This partially answers a question that arose in [T.R. Jensen, B. Toft, Graph coloring problems, Wiley Interscience Series in Discrete Mathematics and Optimization, 1995, p. 204]. We also consider similar problems for bipartite multigraphs.

Keywords: edge-coloring, interval coloring, bipartite graph, bipartite multigraph

\section{Introduction}

In this paper we consider graphs which are finite, undirected, and have no loops or multiple edges and multigraphs which may contain multiple edges but no loops. Let $V(G)$ and $E(G)$ denote the sets of vertices and edges of a multigraph $G$, respectively. For two distinct vertices $u$ and $v$ of a multigraph $G$, let $E(u v)$ denote the set of all edges of $G$ joining $u$ with $v$, and let $\mu(u v)$ denote the number of edges joining $u$ with $v$ (i.e. $\mu(u v)=|E(u v)|)$. The degree of a vertex $v \in V(G)$ is denoted by $d_{G}(v)$ (or $d(v)$ ), the maximum degree of $G$ by $\Delta(G)$, and the edge-chromatic number of $G$ by $\chi^{\prime}(G)$. The terms and concepts that we do not define can be found in [23].

Let $G$ be a connected graph and $V(G)=\left\{v_{1}, \ldots, v_{n}\right\}, n \geq 2$. Let $P\left(v_{i}, v_{j}\right)$ be a simple path joining $v_{i}$ and $v_{j}, V P\left(v_{i}, v_{j}\right)$ and $E P\left(v_{i}, v_{j}\right)$ denote the sets of vertices and edges of

*email: pet_petros@\{ipia.sci.am, ysu.am, yahoo.com\}

†email: hrant@egern.net 
this path, respectively.

A proper edge-coloring of a multigraph $G$ is a coloring of the edges of $G$ such that no two adjacent edges receive the same color. If $\alpha$ is a proper edge-coloring of $G$ and $v \in V(G)$, then $S(v, \alpha)$ denotes the set of colors of edges incident to $v$. A proper edge-coloring of a multigraph $G$ with colors $1, \ldots, t$ is called an interval $t$-coloring if all colors are used, and for any vertex $v$ of $G$, the set $S(v, \alpha)$ is an interval of integers. A multigraph $G$ is interval colorable if it has an interval $t$-coloring for some positive integer $t$. The set of all interval colorable multigraphs is denoted by $\mathfrak{N}$. For a multigraph $G \in \mathfrak{N}$, the least value of $t$ for which $G$ has an interval $t$-coloring is denoted by $w(G)$.

The concept of interval edge-coloring of multigraphs was introduced by Asratian and Kamalian [1, 2]. In [1, 2] they proved that if $G$ is interval colorable, then $\chi^{\prime}(G)=\Delta(G)$. Moreover, if $G$ is $r$-regular, then $G$ has an interval coloring if and only if $G$ has a proper $r$-edge-coloring. This implies that the problem "Is a given $r$-regular $(r \geq 3)$ graph interval colorable or not?" is NP-complete. Asratian and Kamalian also proved [ 1, 2] that if a triangle-free graph $G$ has an interval $t$-coloring, then $t \leq|V(G)|-1$. In [13] Kamalian investigated interval colorings of complete bipartite graphs and trees. In particular, he proved that the complete bipartite graph $K_{m, n}$ has an interval $t$-coloring if and only if $m+n-\operatorname{gcd}(m, n) \leq t \leq m+n-1$, where $\operatorname{gcd}(m, n)$ is the greatest common divisor of $m$ and $n$. In [18] Petrosyan investigated interval colorings of complete graphs and $n$ dimensional cubes. In particular, he proved that if $n \leq t \leq \frac{n(n+1)}{2}$, then the $n$-dimensional cube $Q_{n}$ has an interval $t$-coloring. In [20] Sevast'janov proved that it is an $N P$-complete problem to decide whether a bipartite graph has an interval coloring or not. On the other hand, computer search in [5] showed that the following result holds.

Theorem 1 All bipartite graphs of order at most 14 are interval colorable.

For subcubic bipartite graphs, Hansen proved the following

Theorem 2 [10]. If $G$ is a bipartite graph with $\Delta(G) \leq 3$, then $G \in \mathfrak{N}$ and $w(G) \leq 4$.

For bipartite graphs with maximum degree 4, Giaro proved the following two results:

Theorem 3 [6]. If $G$ is a bipartite graph with $\Delta(G)=4$ and without a vertex of degree 3 , then $G \in \mathfrak{N}$ and $w(G)=4$.

Theorem 4 [6]. The problem of deciding the existence of interval $\Delta(G)$-coloring of a bipartite graph $G$ can be solved in polynomial time if $\Delta(G) \leq 4$ and is NP-complete if $\Delta(G) \geq 5$.

For bipartite graphs where one of the parts is small, the following theorem was proved in $[8]$.

Theorem 5 If $G$ is a bipartite graph with a bipartition $(U, V)$ and $\min \{|U|,|V|\} \leq 3$, then $G \in \mathfrak{N}$. 
Also, it is known that all regular bipartite graphs [1, 2], doubly convex bipartite graphs [ 3, 14], grids [ 7], outerplanar bipartite graphs [ 9], (2,b)-biregular bipartite graphs [ [11, 15, 16] and some classes of (3,4)-biregular bipartite graphs [4, 19, 24, have interval colorings. However, there are bipartite graphs which have no interval colorings. First example of a bipartite graph that is not interval colorable was obtained by Mirumyan [ 17] in 1989, but it was not published. The graph which was found by Mirumyan has 19 vertices and maximum degree 15. First published example was given by Sevast'janov [ 20] and it has 28 vertices and maximum degree 21 (see Fig. 11). Other examples were obtained by Erdős (27 vertices and maximum degree 13), by Hertz and de Werra (21 vertices and maximum degree 14), and by Malafiejski (19 vertices and maximum degree 15). In [12], Jensen and Toft posed the following question:

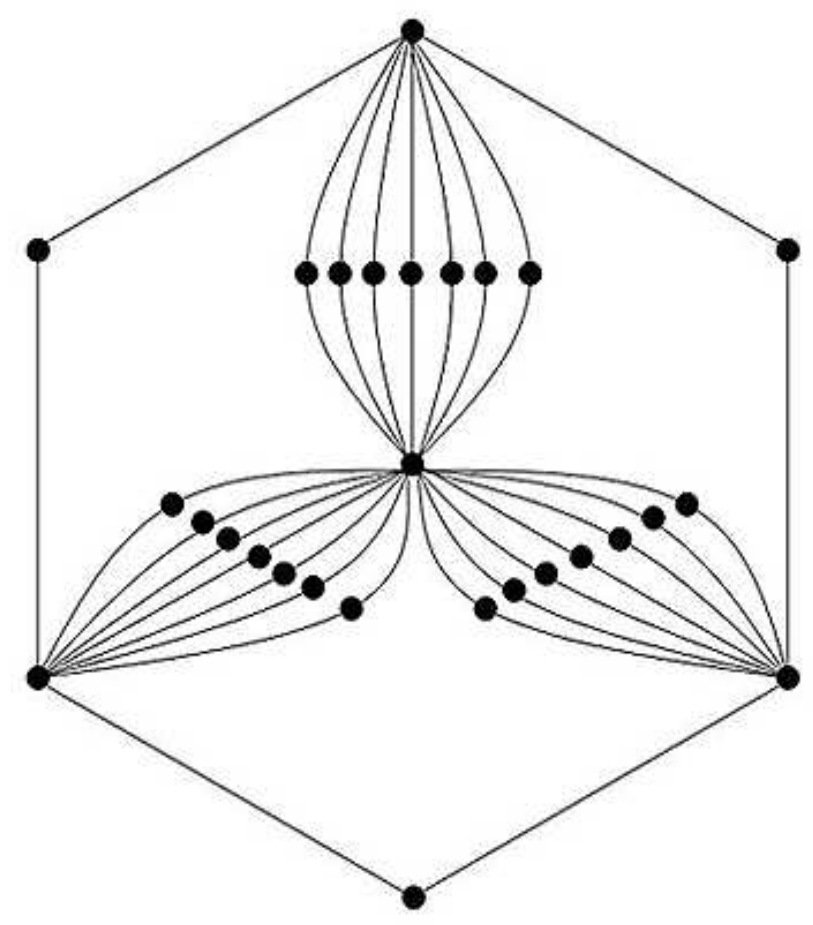

Figure 1. The Sevast'janov graph.

Problem 1 Is there a bipartite graph $G$ with $4 \leq \Delta(G) \leq 12$ and $G \notin \mathfrak{N}$ ?

In the present paper we describe some methods for constructing of interval non-edgecolorable bipartite graphs. In particular, by these methods, we construct two bipartite graphs $G$ and $H$ with $\Delta(G)=11, \Delta(H)=12$ which have no interval coloring. This partially answers a question of Jensen and Toft. In this paper we also consider similar problems for bipartite multigraphs. 


\section{Interval non-edge-colorable bipartite graphs}

\subsection{Counterexamples by fat triangles}

In 1949 Shannon [ 21] proved that $\chi^{\prime}(G) \leq\left\lfloor\frac{3}{2} \Delta(G)\right\rfloor$ for any multigraph $G$. Also, he showed that this upper bound is sharp for special multigraphs which are called fat triangles. The fat triangle is a multigraph with three vertices $x, y, z$ and $r$ edges between each pair of vertices, that is, $\mu(x y)=\mu(y z)=\mu(x z)=r$. Later, Vizing [22] proved that if a multigraph $G$ has $\chi^{\prime}(G)=\left\lfloor\frac{3}{2} \Delta(G)\right\rfloor$ and $\Delta(G) \geq 4$, then $G$ has a fat triangle as a subgraph. In this paragraph we use fat triangles for constructing of interval non-edgecolorable bipartite graphs. First note that the graph obtained by subdividing every edge of a fat triangle is bipartite. Moreover, a new graph obtained from the subdivided graph by connecting every inserted vertex to a new vertex is also bipartite. Now let us define the graph $\Delta_{r, s, t}(1 \leq r \leq s \leq t)$ as follows:

$$
\begin{gathered}
V\left(\Delta_{r, s, t}\right)=\{v, x, y, z\} \cup\left\{a_{1}, \ldots, a_{r}, b_{1}, \ldots, b_{s}, c_{1}, \ldots, c_{t}\right\}, \\
E\left(\Delta_{r, s, t}\right)=\left\{v a_{i}, x a_{i}, y a_{i}: 1 \leq i \leq r\right\} \cup\left\{v b_{j}, x b_{j}, z b_{j}: 1 \leq j \leq s\right\} \cup\left\{v c_{k}, y c_{k}, z c_{k}: 1 \leq\right. \\
k \leq t\} .
\end{gathered}
$$

Clearly, $\Delta_{r, s, t}$ is a connected bipartite graph with $\left|V\left(\Delta_{r, s, t}\right)\right|=r+s+t+4, d(x)=r+s$, $d(y)=r+t, d(z)=s+t$, and $\Delta\left(\Delta_{r, s, t}\right)=r+s+t$. Note that our $\Delta_{r, s, t}$ graphs generalize Malafiejski's rosettes $M_{k}$ given in [8], since $M_{k}=\Delta_{k, k, k}$ for any $k \in \mathbf{N}$.

Theorem 6 If $r \geq 5$, then $\Delta_{r, s, t} \notin \mathfrak{N}$.

Proof. Suppose, to the contrary, that the graph $\Delta_{r, s, t}$ has an interval $q$-coloring $\alpha$ for some $q \geq r+s+t$.

Consider the vertex $v$. Let $u$ and $w$ be two vertices adjacent to $v$ such that $\alpha(v u)=$ $\min S(v, \alpha)=p$ and $\alpha(v w)=\max S(v, \alpha)=p+r+s+t-1$. By the construction of $\Delta_{r, s, t}$, there is a path $P(u, w)$ in $\Delta_{r, s, t}-v$ of length two joining $u$ with $w$, where

$$
P(u, w)=\left(u, u v^{\prime}, v^{\prime}, v^{\prime} w, w\right) .
$$

Since $d(u)=3$ and $d\left(v^{\prime}\right) \leq s+t$, we have

$$
\begin{gathered}
\alpha\left(u v^{\prime}\right) \leq p+d(u)-1=p+2 \text { and thus } \\
\alpha\left(v^{\prime} w\right) \leq p+2+d\left(v^{\prime}\right)-1=p+1+s+t .
\end{gathered}
$$

On the other hand, since $d(w)=3$, we have

$$
p+r+s+t-1=\alpha(v w)=\max S(v, \alpha) \leq p+1+s+t+d(w)-1=p+s+t+3 .
$$

Hence, $r \leq 4$, which is a contradiction. 


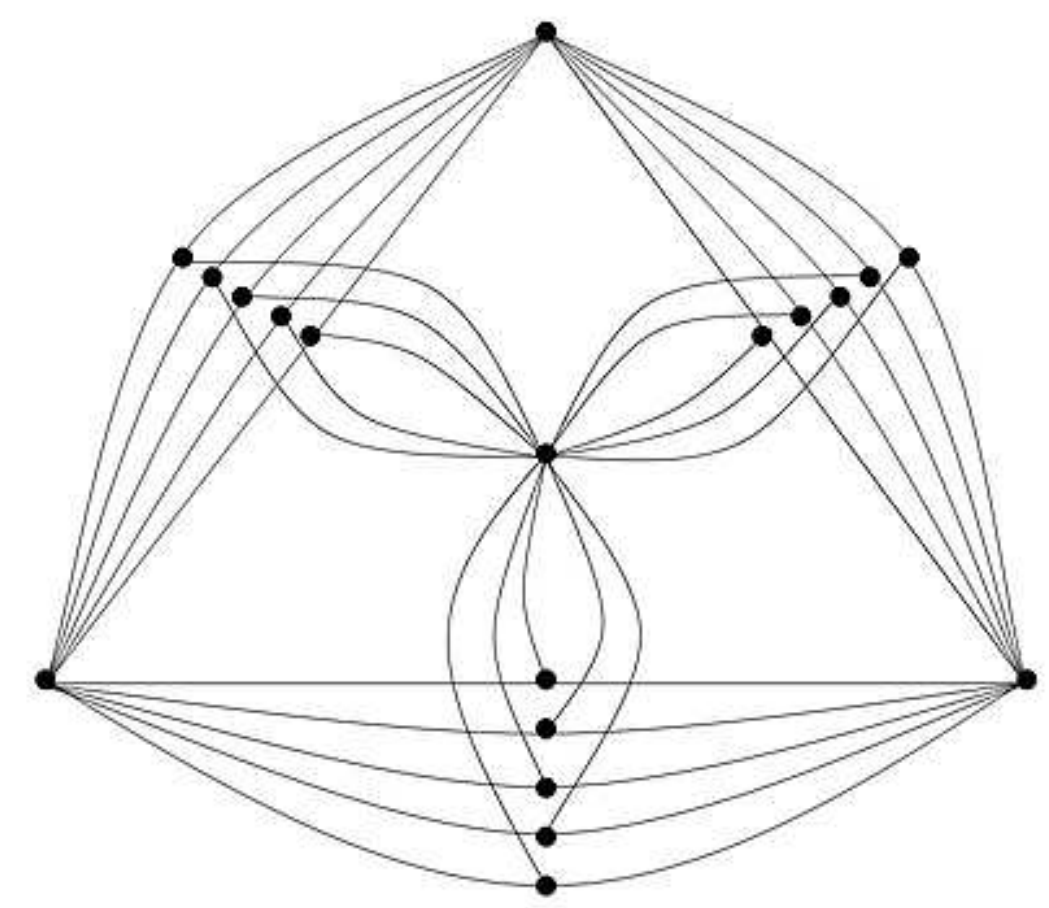

Figure 2. The graph $\Delta_{5,5,5}$.

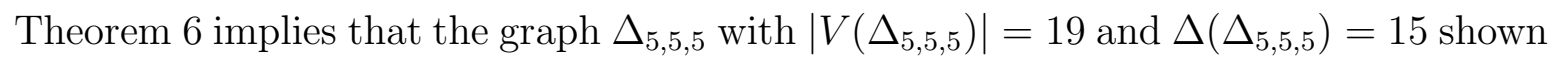
in Fig. 2 has no interval coloring. In fact, this is the example of an interval non-edgecolorable bipartite graph that first was constructed by Mirumyan. This example first appeared in [3] and [8], and currently is known as Malafiejski's rosette $M_{5}$.

Corollary 7 For any positive integer $\Delta \geq 15$, there is a connected bipartite graph $G$ with $G \notin \mathfrak{N}$ and $\Delta(G)=\Delta$.

\subsection{Counterexamples by finite projective planes}

In this paragraph we use finite projective planes for constructing of interval non-edgecolorable bipartite graphs. A finite projective plane $\pi(n)$ of order $n(n \geq 2)$ has $n^{2}+n+1$ points and $n^{2}+n+1$ lines, and satisfies the following properties:

$\mathbf{P 1}$ any two points determine a line;

P2 any two lines determine a point;

P3 every point is incident to $n+1$ lines;

$\mathbf{P} 4$ every line is incident to $n+1$ points. 
Let $\left\{1, \ldots, n^{2}+n+1\right\}$ be the set of points and $L$ be the set of lines of $\pi(n)$. Define the graph $\operatorname{Erd}\left(r_{1}, \ldots, r_{n^{2}+n+1}\right)\left(r_{1} \geq \ldots \geq r_{n^{2}+n+1} \geq 1\right)$ as follows:

$$
\begin{gathered}
V\left(\operatorname{Erd}\left(r_{1}, \ldots, r_{n^{2}+n+1}\right)\right)=\{u\} \cup\left\{1, \ldots, n^{2}+n+1\right\} \\
\cup\left\{v_{1}^{\left(l_{i}\right)}, \ldots, v_{r_{i}}^{\left(l_{i}\right)}: l_{i} \in L, 1 \leq i \leq n^{2}+n+1\right\}, \\
E\left(\operatorname{Erd}\left(r_{1}, \ldots, r_{n^{2}+n+1}\right)\right)=\left\{u v_{1}^{\left(l_{i}\right)}, \ldots, u v_{r_{i}}^{\left(l_{i}\right)}: l_{i} \in L, 1 \leq i \leq n^{2}+n+1\right\} \cup \\
\bigcup_{i=1}^{n^{2}+n+1}\left\{v_{1}^{\left(l_{i}\right)} k, \ldots, v_{r_{i}}^{\left(l_{i}\right)} k: l_{i} \in L, k \in l_{i}, 1 \leq k \leq n^{2}+n+1\right\} .
\end{gathered}
$$

Clearly, $\operatorname{Erd}\left(r_{1}, \ldots, r_{n^{2}+n+1}\right)$ is a connected bipartite graph with $\Delta\left(\operatorname{Erd}\left(r_{1}, \ldots, r_{n^{2}+n+1}\right)\right)=$ $\sum_{i=1}^{n^{2}+n+1} r_{i}$ and $\left|V\left(\operatorname{Erd}\left(r_{1}, \ldots, r_{n^{2}+n+1}\right)\right)\right|=\sum_{i=1}^{n^{2}+n+1} r_{i}+n^{2}+n+2$. Note that the graph $\operatorname{Erd}(1,1,1,1,1,1,1,1,1,1,1,1,1)$ was described by Erdös in 1991 [12]. This graph has 27 vertices and maximum degree 13 .

Theorem 8 If $\sum_{i=n+2}^{n^{2}+n+1} r_{i}>2(n+1)$, then $\operatorname{Erd}\left(r_{1}, \ldots, r_{n^{2}+n+1}\right) \notin \mathfrak{N}$.

Proof. Suppose, to the contrary, that the graph $\operatorname{Erd}\left(r_{1}, \ldots, r_{n^{2}+n+1}\right)$ has an interval $t$-coloring $\alpha$ for some $t \geq \sum_{i=1}^{n^{2}+n+1} r_{i}$.

Consider the vertex $u$. Let $v_{p}^{\left(l_{i_{0}}\right)}$ and $v_{q}^{\left(l_{j_{0}}\right)}$ be two vertices adjacent to $u$ such that $\alpha\left(u v_{p}^{\left(l_{i_{0}}\right)}\right)=\min S(u, \alpha)=s$ and $\alpha\left(u v_{q}^{\left(l_{j_{0}}\right)}\right)=\max S(u, \alpha)=s+\sum_{i=1}^{n^{2}+n+1} r_{i}-1$.

If $l_{i_{0}}=l_{j_{0}}$, then, by the construction of $\operatorname{Erd}\left(r_{1}, \ldots, r_{n^{2}+n+1}\right)$, there exists $k_{0}$ such that $k_{0} v_{p}^{\left(l_{i_{0}}\right)}, k_{0} v_{q}^{\left(l_{j_{0}}\right)} \in E\left(\operatorname{Erd}\left(r_{1}, \ldots, r_{n^{2}+n+1}\right)\right)$. If $l_{i_{0}} \neq l_{j_{0}}$, then, by the construction of $\operatorname{Erd}\left(r_{1}, \ldots, r_{n^{2}+n+1}\right)$ and the property P2, there exists $k_{0}$ such that $k_{0} v_{p}^{\left(l_{i_{0}}\right)}, k_{0} v_{q}^{\left(l_{j_{0}}\right)} \in$ $E\left(\operatorname{Erd}\left(r_{1}, \ldots, r_{n^{2}+n+1}\right)\right)$.

By the construction of $\operatorname{Erd}\left(r_{1}, \ldots, r_{n^{2}+n+1}\right)$ and properties $\mathrm{P} 3$ and $\mathrm{P} 4$, we have $d\left(v_{p}^{\left(l_{i_{0}}\right)}\right)=$ $d\left(v_{q}^{\left(l_{j_{0}}\right)}\right)=n+2$ and

$$
\begin{aligned}
& \alpha\left(k_{0} v_{p}^{\left(l_{i_{0}}\right)}\right) \leq s+d\left(v_{p}^{\left(l_{i_{0}}\right)}\right)-1=s+n+1 \text { and thus } \\
& \alpha\left(k_{0} v_{q}^{\left(l_{j_{0}}\right)}\right) \leq s+n+1+d\left(k_{0}\right)-1 \leq s+n+\sum_{i=1}^{n+1} r_{i} .
\end{aligned}
$$

This implies that

$$
s+\sum_{i=1}^{n^{2}+n+1} r_{i}-1=\alpha\left(u v_{q}^{\left(l_{j_{0}}\right)}\right)=\max S(u, \alpha) \leq s+n+\sum_{i=1}^{n+1} r_{i}+d\left(v_{q}^{\left(l_{j_{0}}\right)}\right)-1=s+2 n+1+\sum_{i=1}^{n+1} r_{i} .
$$

Hence, $\sum_{i=n+2}^{n^{2}+n+1} r_{i} \leq 2(n+1)$, which is a contradiction. 


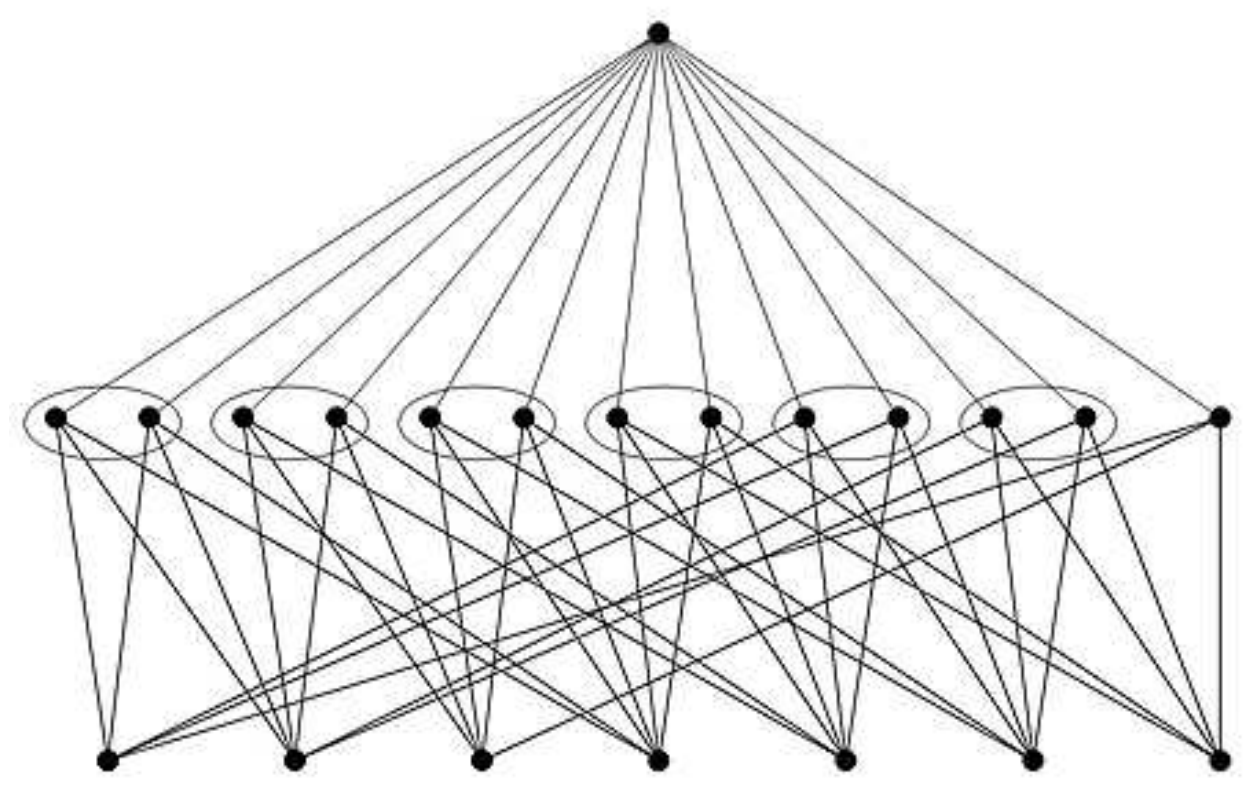

Figure 3. The graph $\operatorname{Erd}(2,2,2,2,2,2,1)$.

Corollary 9 For any positive integer $\Delta \geq 13$, there is a connected bipartite graph $G$ with $G \notin \mathfrak{N}$ and $\Delta(G)=\Delta$.

Theorem 8 implies that the graph $\operatorname{Erd}(2,2,2,2,2,2,1)$ with $|\operatorname{V}(\operatorname{Erd}(2,2,2,2,2,2,1))|=$ 21 and $\Delta(\operatorname{Erd}(2,2,2,2,2,2,1))=13$ shown in Fig. 3 has no interval coloring. Also, Theorem 8 implies that the graph $\operatorname{Erd}(2,2,2,2,2,2,2)$ with $\mid \operatorname{VErd}(2,2,2,2,2,2,2)) \mid=$ 22 and $\Delta(\operatorname{Erd}(2,2,2,2,2,2,1))=14$ has no interval coloring. In the next section we show that there is a connected bipartite graph $G$ with $|V(G)|=21$ and $\Delta(G)=14$ which is not interval colorable.

\subsection{Counterexamples by trees}

Let $T$ be a tree and $V(T)=\left\{v_{1}, \ldots, v_{n}\right\}, n \geq 2$. For a simple path $P\left(v_{i}, v_{j}\right)$, define $L\left(v_{i}, v_{j}\right)$ as follows:

$$
L\left(v_{i}, v_{j}\right)=\left|E P\left(v_{i}, v_{j}\right)\right|+\left|\left\{u w: u w \in E(T), u \in V P\left(v_{i}, v_{j}\right), w \notin V P\left(v_{i}, v_{j}\right)\right\}\right| .
$$

Define:

$$
M(T)=\max _{1 \leq i \leq n, 1 \leq j \leq n} L\left(v_{i}, v_{j}\right) .
$$

In [14], Kamalian proved the following result. 
Theorem 10 If $T$ is a tree, then $T$ has an interval $t$-coloring if and only if $\Delta(T) \leq t \leq$ $M(T)$.

Now let $T$ be a tree in which the distance between any two pendant vertices is even and $F(T)=\left\{v: v \in V(T) \wedge d_{T}(v)=1\right\}$.

Let us define the graph $\widetilde{T}$ as follows:

$$
V(\widetilde{T})=V(T) \cup\{u\}, u \notin V(T), E(\widetilde{T})=E(T) \cup\{u v: v \in F(T)\} .
$$

Clearly, $\widetilde{T}$ is a connected bipartite graph with $\Delta(\widetilde{T})=|F(T)|$.

Theorem 11 If $T$ is a tree in which the distance between any two pendant vertices is even and $|F(T)|>M(T)+2$, then $\widetilde{T} \notin \mathfrak{N}$.

Proof. Suppose, to the contrary, that $\widetilde{T}$ has an interval $t$-coloring $\alpha$ for some $t \geq|F(T)|$.

Consider the vertex $u$. Let $v$ and $v^{\prime}$ be two vertices adjacent to $u$ such that $\alpha(u v)=$ $\min S(u, \alpha)=s$ and $\alpha\left(u v^{\prime}\right)=\max S(u, \alpha)=s+|F(T)|-1$. Since $\widetilde{T}-u$ is a tree, there is a unique path $P\left(v, v^{\prime}\right)$ in $\widetilde{T}-u$ joining $v$ with $v^{\prime}$, where

$$
P\left(v, v^{\prime}\right)=\left(v_{1}, e_{1}, v_{2}, \ldots, v_{i}, e_{i}, v_{i+1}, \ldots, v_{k}, e_{k}, v_{k+1}\right), v_{1}=v, v_{k+1}=v^{\prime}
$$

Note that

$$
\alpha\left(v_{i} v_{i+1}\right) \leq s+1+\sum_{j=1}^{i}\left(d_{T}\left(v_{j}\right)-1\right) \text { for } 1 \leq i \leq k
$$

From this, we have

$$
\alpha\left(v_{k} v_{k+1}\right)=\alpha\left(v_{k} v^{\prime}\right) \leq s+1+\sum_{j=1}^{k}\left(d_{T}\left(v_{j}\right)-1\right)=s+L\left(v, v^{\prime}\right) \leq s+M(T)
$$

Hence

$$
s+|F(T)|-1=\max S(u, \alpha)=\alpha\left(u v^{\prime}\right) \leq s+1+M(T) \text { and thus }|F(T)| \leq M(T)+2,
$$

which is a contradiction.

Now let us consider the tree $T$ shown in Fig. 4,

Since $M(T)=11$ and $|F(T)|=14$, the graph $\widetilde{T}$ with $|V(\widetilde{T})|=21$ and $\Delta(\widetilde{T})=14$ has no interval coloring. Our constructions by trees generalize Hertz's graphs $H_{p, q}$ given in [8]. Moreover, the aforementioned example obtained by the method described above is smaller than the smallest Hertz's graph $H_{7,2}$. 


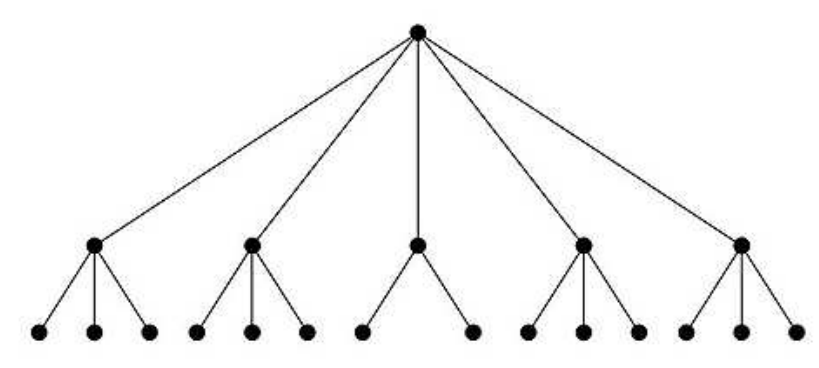

Figure 4. The tree $T$.

\subsection{Counterexamples by subdivisions}

In this section we also need a definition of the interval of positive integers. For positive integers $a$ and $b$, we denote by $[a, b]$, the set of all positive integers $c$ with $a \leq c \leq b$.

Let $G$ be a graph and $V(G)=\left\{v_{1}, \ldots, v_{n}\right\}$. Define graphs $S(G)$ and $\widehat{G}$ as follows:

$$
\begin{gathered}
V(S(G))=\left\{v_{1}, \ldots, v_{n}\right\} \cup\left\{w_{i j}: v_{i} v_{j} \in E(G)\right\}, \\
E(S(G))=\left\{v_{i} w_{i j}, v_{j} w_{i j}: v_{i} v_{j} \in E(G)\right\}, \\
V(\widehat{G})=V(S(G)) \cup\{u\}, u \notin V(S(G)), E(\widehat{G})=E(S(G)) \cup\left\{u w_{i j}: v_{i} v_{j} \in E(G)\right\} .
\end{gathered}
$$

In other words, $S(G)$ is the graph obtained by subdividing every edge of $G$, and $\widehat{G}$ is the graph obtained from $S(G)$ by connecting every inserted vertex to a new vertex $u$. Clearly, $S(G)$ and $\widehat{G}$ are bipartite graphs.

Proposition 12 If $G$ is a bipartite graph and $G \in \mathfrak{N}$, then $S(G) \in \mathfrak{N}$.

Proof. Let $G$ be a bipartite graph with a bipartition $(U, V)$, where $U=\left\{u_{1}, \ldots, u_{r}\right\}$, $V=\left\{v_{1}, \ldots, v_{s}\right\}$. Also, let $\alpha$ be an interval $t$-coloring of the graph $G$.

Define an edge-coloring $\beta$ of the graph $S(G)$ as follows:

$$
\beta\left(u_{i} w_{i j}\right)=\alpha\left(u_{i} v_{j}\right) \text { and } \beta\left(v_{j} w_{i j}\right)=\alpha\left(u_{i} v_{j}\right)+1 \text { for every } u_{i} v_{j} \in E(G) .
$$

It is easy to see that $\beta$ is an interval $(t+1)$-coloring of the graph $S(G)$.

In [11, 15, 16], it was proved that if $G$ is a regular graph, then $S(G) \in \mathfrak{N}$. It would be interesting to generalize the last two statements to general graphs. In other words, we would like to suggest the following

Conjecture 13 If $G$ is a simple graph and $G \in \mathfrak{N}$, then $S(G) \in \mathfrak{N}$.

Theorem 14 If $G$ is a connected graph and 


$$
|E(G)|>1+\max _{P \in \mathbf{P}} \sum_{v \in V(P)}\left(d_{\widehat{G}}(v)-1\right),
$$

where $\mathbf{P}$ is a set of all shortest paths in $S(G)$ connecting vertices $w_{i j}$, then $\widehat{G} \notin \mathfrak{N}$.

Proof. Suppose, to the contrary, that $\widehat{G}$ has an interval $t$-coloring $\alpha$ for some $t \geq|E(G)|$.

Consider the vertex $u$. Let $w$ and $w^{\prime}$ be two vertices adjacent to $u$ such that $\alpha(u w)=$ $\min S(u, \alpha)=s$ and $\alpha\left(u w^{\prime}\right)=\max S(u, \alpha)=s+|E(G)|-1$. Since $\widehat{G}-u$ is isomorphic to $S(G)$ and connected, there is a shortest path $P\left(w, w^{\prime}\right)$ in $\widehat{G}-u$ joining $w$ with $w^{\prime}$, where

$$
P\left(w, w^{\prime}\right)=\left(v_{1}, e_{1}, v_{2}, \ldots, v_{i}, e_{i}, v_{i+1}, \ldots, v_{k}, e_{k}, v_{k+1}\right), v_{1}=w, v_{k+1}=w^{\prime} .
$$

Note that

$$
\alpha\left(v_{i} v_{i+1}\right) \leq s+\sum_{j=1}^{i}\left(d_{\widehat{G}}\left(v_{j}\right)-1\right) \text { for } 1 \leq i \leq k
$$

and

$$
\alpha\left(v_{k+1} u\right)=\alpha\left(w^{\prime} u\right) \leq s+\sum_{j=1}^{k+1}\left(d_{\widehat{G}}\left(v_{j}\right)-1\right)
$$

Hence

$s+|E(G)|-1=\max S(u, \alpha)=\alpha\left(u w^{\prime}\right) \leq s+\sum_{j=1}^{k+1}\left(d_{\widehat{G}}\left(v_{j}\right)-1\right) \leq s+\max _{P \in \mathbf{P}} \sum_{v \in V(P)}\left(d_{\widehat{G}}(v)-1\right)$

and thus

$$
|E(G)| \leq 1+\max _{P \in \mathbf{P}} \sum_{v \in V(P)}\left(d_{\widehat{G}}(v)-1\right),
$$

which is a contradiction.

Corollary 15 If $n \geq 7$, then $\widehat{K}_{n} \notin \mathfrak{N}$.

Corollary 16 If $m n-m-n>5$, then $\widehat{K}_{m, n} \notin \mathfrak{N}$.

Now we show that the graph $\widehat{K}_{3,4}$ shown in Fig. 5 has no interval coloring.

Theorem $17 \widehat{K}_{3,4} \notin \mathfrak{N}$.

Proof. Let $V\left(\widehat{K}_{3,4}\right)=\left\{u, v_{1}, v_{2}, v_{3}, v_{4}, v_{5}, v_{6}, v_{7}\right\} \cup\left\{w_{i j}: 1 \leq i \leq 3,4 \leq j \leq 7\right\}$ and $E\left(\widehat{K}_{3,4}\right)=\left\{v_{i} w_{i j}, v_{j} w_{i j}, u w_{i j}: 1 \leq i \leq 3,4 \leq j \leq 7\right\}$.

Suppose that $\widehat{K}_{3,4}$ has an interval $t$-coloring $\alpha$ for some $t \geq 12$.

Consider the vertex $u$. Let $w_{i_{0} j_{0}}$ and $w_{i_{1} j_{1}}$ be two vertices adjacent to $u$ such that $\alpha\left(u w_{i_{0} j_{0}}\right)=\min S(u, \alpha)=s$ and $\alpha\left(u w_{i_{1} j_{1}}\right)=\max S(u, \alpha)=s+11$. We consider two cases.

Case 1: $i_{0}=i_{1}$ or $j_{0}=j_{1}$.

If $i_{0}=i_{1}$, then $v_{i_{0}} w_{i_{0} j_{0}}, v_{i_{0}} w_{i_{0} j_{1}} \in E\left(\widehat{K}_{3,4}\right)$. This implies that 


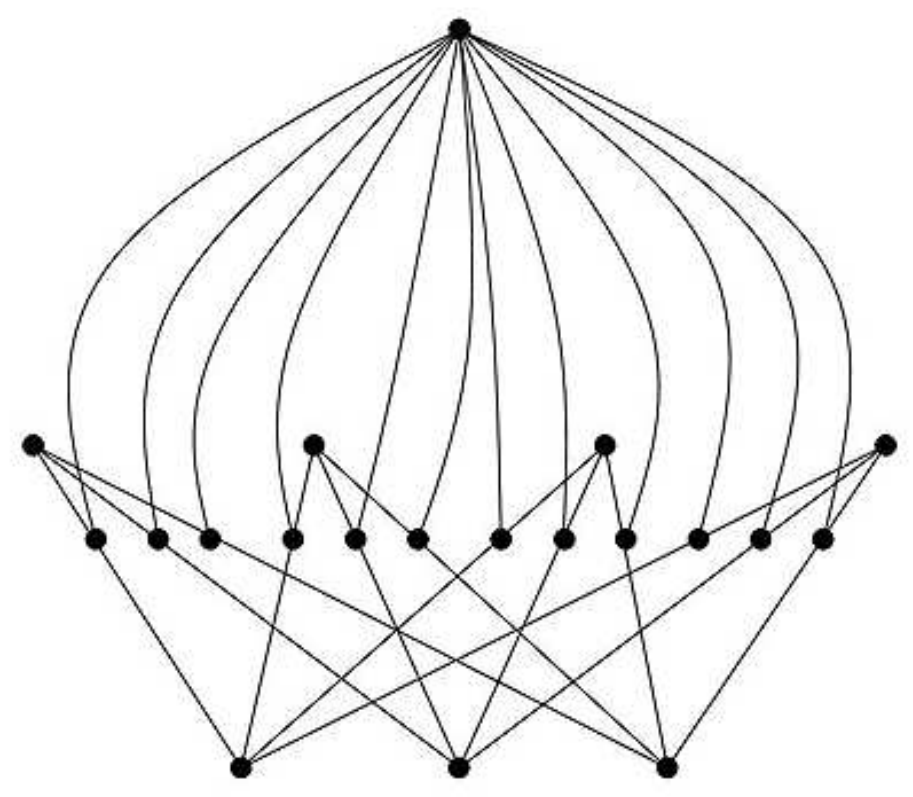

Figure 5. The graph $\widehat{K}_{3,4}$.

$$
\alpha\left(v_{i_{0}} w_{i_{0} j_{0}}\right) \leq s+2 \text { and } \alpha\left(v_{i_{0}} w_{i_{0} j_{1}}\right) \leq s+5
$$

Hence,

$$
s+11=\max S(u, \alpha)=\alpha\left(u w_{i_{0} j_{1}}\right) \leq s+7,
$$

which is impossible.

If $j_{0}=j_{1}$, then $v_{j_{0}} w_{i_{0} j_{0}}, v_{j_{0}} w_{i_{1} j_{0}} \in E\left(\widehat{K}_{3,4}\right)$. This implies that

$$
\alpha\left(v_{j_{0}} w_{i_{0} j_{0}}\right) \leq s+2 \text { and } \alpha\left(v_{j_{0}} w_{i_{1} j_{0}}\right) \leq s+4 .
$$

Hence,

$$
s+11=\max S(u, \alpha)=\alpha\left(u w_{i_{1} j_{0}}\right) \leq s+6,
$$

which is impossible.

Case 2: $i_{0} \neq i_{1}$ and $j_{0} \neq j_{1}$.

In this case the edges $v_{i_{0}} v_{j_{0}}$ and $v_{i_{1}} v_{j_{1}}$ are independent in $K_{3,4}$. Clearly, any two independent edges in $K_{3,4}$ lie on the cycle of a length four. Hence, there is a cycle $C=w_{i_{0} j_{0}} v_{j_{0}} w_{i_{1} j_{0}} v_{i_{1}} w_{i_{1} j_{1}} v_{j_{1}} w_{i_{0} j_{1}} v_{i_{0}} w_{i_{0} j_{0}}$ in $\widehat{K}_{3,4}$, which is consists of paths $P$ and $Q$, where

$$
P=\left(w_{i_{0} j_{0}}, v_{j_{0}} w_{i_{0} j_{0}}, v_{j_{0}}, v_{j_{0}} w_{i_{1} j_{0}}, w_{i_{1} j_{0}}, v_{i_{1}} w_{i_{1} j_{0}}, v_{i_{1}}, v_{i_{1}} w_{i_{1} j_{1}}, w_{i_{1} j_{1}}\right)
$$

and 


$$
Q=\left(w_{i_{0} j_{0}}, v_{i_{0}} w_{i_{0} j_{0}}, v_{i_{0}}, v_{i_{0}} w_{i_{0} j_{1}}, w_{i_{0} j_{1}}, v_{j_{1}} w_{i_{0} j_{1}}, v_{j_{1}}, v_{j_{1}} w_{i_{1} j_{1}}, w_{i_{1} j_{1}}\right)
$$

If $\alpha\left(v_{j_{0}} w_{i_{0} j_{0}}\right)=s+1$, then, by considering the path $P$, we have $\alpha\left(v_{i_{1}} w_{i_{1} j_{1}}\right) \leq s+8$ and $\max S\left(w_{i_{1} j_{1}}, \alpha\right) \leq s+10$, a contradiction.

If $\alpha\left(v_{i_{0}} w_{i_{0} j_{0}}\right)=s+1$, then, by considering the path $Q$, we have $\alpha\left(v_{j_{1}} w_{i_{1} j_{1}}\right) \leq s+8$ and $\max S\left(w_{i_{1} j_{1}}, \alpha\right) \leq s+10$, a contradiction.

Hence, $\alpha\left(v_{j_{0}} w_{i_{0} j_{0}}\right)=\alpha\left(v_{i_{0}} w_{i_{0} j_{0}}\right)=s+2$, which is a contradiction.

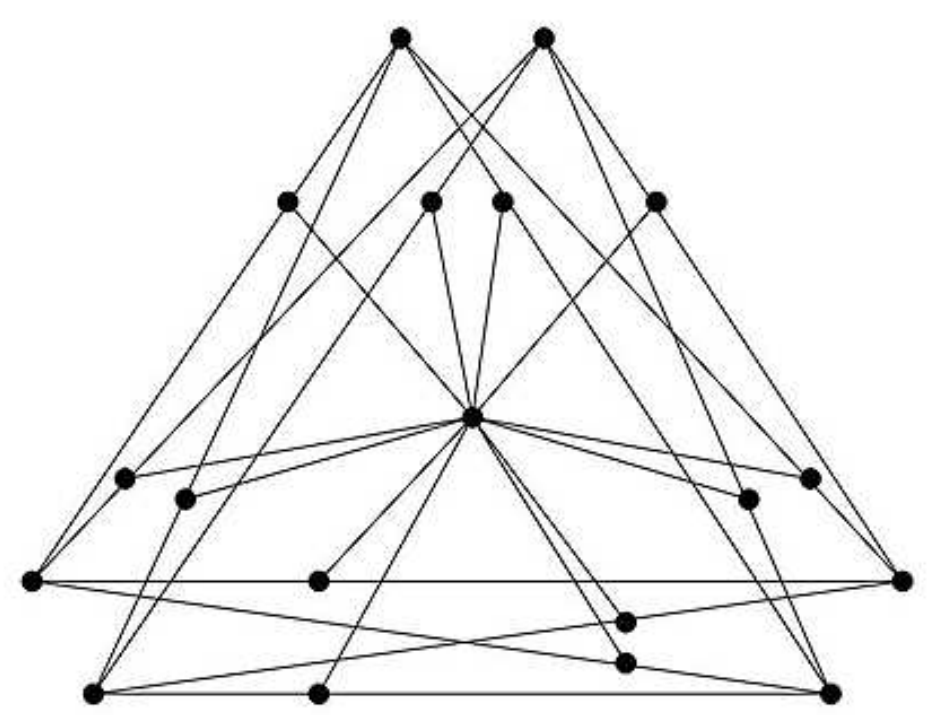

Figure 6. The graph $\widehat{K}_{2,2,2}$.

Note that the graph $\widehat{K}_{3,4}$ has 20 vertices and maximum degree 12 . Now we show that there is a connected bipartite graph $G$ with $|V(G)|=19$ and $\Delta(G)=12$ which is not interval colorable. Let $K_{2,2,2}$ be a complete 3 -partite graph with two vertices in each part. Then the graph $\widehat{K}_{2,2,2}$ shown in Fig. [6 is not interval colorable.

Theorem $18 \widehat{K}_{2,2,2} \notin \mathfrak{N}$.

Proof. Let $V\left(\widehat{K}_{2,2,2}\right)=\left\{u, v_{1}, v_{2}, v_{3}, v_{4}, v_{5}, v_{6}\right\} \cup\left\{w_{i j}: 1 \leq i<j \leq 6,(i, j) \notin\{(1,2),(3,4)\right.$, $(5,6)\}\}$ and $E\left(\widehat{K}_{2,2,2}\right)=\left\{v_{i} w_{i j}, v_{j} w_{i j}, u w_{i j}: 1 \leq i<j \leq 6,(i, j) \notin\{(1,2),(3,4),(5,6)\}\right\}$.

Suppose that $\widehat{K}_{2,2,2}$ has an interval $t$-coloring $\alpha$ for some $t \geq 12$.

Consider the vertex $u$. Let $w_{i_{0} j_{0}}$ and $w_{i_{1} j_{1}}$ be two vertices adjacent to $u$ such that $\alpha\left(u w_{i_{0} j_{0}}\right)=\min S(u, \alpha)=s$ and $\alpha\left(u w_{i_{1} j_{1}}\right)=\max S(u, \alpha)=s+11$. By the symmetry of the graph $\widehat{K}_{2,2,2}$, we may assume that $\left(i_{0}, j_{0}\right)=(1,6)$. We consider two cases.

Case 1: $v_{1} v_{6}$ and $v_{i_{1}} v_{j_{1}}$ are adjacent in $K_{2,2,2}$. 
By the symmetry of the graph $\widehat{K}_{2,2,2}$, it suffices to consider $\left(i_{1}, j_{1}\right)=(1,4)$ and $\left(i_{1}, j_{1}\right)=$ $(1,5)$.

If $\alpha\left(u w_{14}\right)=s+11$ or $\alpha\left(u w_{15}\right)=s+11$, then $\alpha\left(v_{1} w_{16}\right) \leq s+2$ and $\alpha\left(v_{1} w_{1 j_{1}}\right) \leq s+5$. Hence, $\alpha\left(u w_{1 j_{1}}\right) \leq s+7$, which is impossible.

Case 2: $v_{1} v_{6}$ and $v_{i_{1}} v_{j_{1}}$ are independent in $K_{2,2,2}$.

By the symmetry of the graph $\widehat{K}_{2,2,2}$, it suffices to consider $\left(i_{1}, j_{1}\right)=(4,5)$ and $\left(i_{1}, j_{1}\right)=$ $(2,5)$.

If $\alpha\left(u w_{45}\right)=s+11$, then either $\alpha\left(v_{1} w_{16}\right)=s+1$ or $\alpha\left(v_{1} w_{16}\right)=s+2$, and in both cases the colors of all edges along the cycle $C=w_{16} v_{1} w_{15} v_{5} w_{45} v_{4} w_{46} v_{6} w_{16}$ are known. This implies that $\alpha\left(v_{1} w_{14}\right) \leq s+4$, but $\alpha\left(v_{4} w_{14}\right) \geq s+7$, which is a contradiction.

If $\alpha\left(u w_{25}\right)=s+11$, then, by the symmetry of the graph $\widehat{K}_{2,2,2}$, we may assume that $\alpha\left(v_{1} w_{16}\right)=s+1$. Clearly, in this case the colors of all edges along the cycle $C=w_{16} v_{1} w_{15} v_{5} w_{25} v_{2} w_{26} v_{6} w_{16}$ are known. This implies that $\alpha\left(u w_{26}\right)=s+6$. By the symmetry of the graph $\widehat{K}_{2,2,2}$, we may assume that $\alpha\left(v_{1} w_{13}\right)=s+2$ and $\alpha\left(v_{1} w_{14}\right)=s+3$. It is easy to see that $\alpha\left(u w_{13}\right)=s+1$. Hence, $\alpha\left(v_{3} w_{13}\right)=s+3$ and taking into account that $\alpha\left(v_{2} w_{25}\right)=s+10$, we have $\alpha\left(v_{3} w_{23}\right)=s+6$. This implies that $\alpha\left(v_{3} w_{35}\right) \leq s+5$. On the other hand, since $\alpha\left(v_{5} w_{25}\right)=s+9$, we have $\alpha\left(v_{5} w_{35}\right) \geq s+7$ and thus $\alpha\left(u w_{35}\right)=$ $s+6=\alpha\left(u w_{26}\right)$, which is a contradiction.

Also, we investigate bipartite graphs which are close to the graph $\widehat{K}_{3,4}$. In particular, we observe that the graph obtained from $\widehat{K}_{3,4}$ by deleting any edge incident to the vertex of maximum degree is not interval colorable, too. Let $\widehat{K}_{3,4}^{\prime}$ be a graph obtained from $\widehat{K}_{3,4}$ by deleting any edge incident to the vertex $u$. Clearly, $\widehat{K}_{3,4}^{\prime}$ has 20 vertices and maximum degree 11.

Theorem $19 \widehat{K}_{3,4}^{\prime} \notin \mathfrak{N}$.

Proof. Let $V\left(\widehat{K}_{3,4}^{\prime}\right)=V\left(\widehat{K}_{3,4}\right)$ and $E\left(\widehat{K}_{3,4}^{\prime}\right)=E\left(\widehat{K}_{3,4}\right) \backslash u w_{l_{0} m_{0}}$.

Suppose that $\widehat{K}_{3,4}^{\prime}$ has an interval $t$-coloring $\alpha$ for some $t \geq 11$.

Consider the vertex $u$. Let $w_{i_{0} j_{0}}$ and $w_{i_{1} j_{1}}$ be two vertices adjacent to $u$ such that $\alpha\left(u w_{i_{0} j_{0}}\right)=\min S(u, \alpha)=s$ and $\alpha\left(u w_{i_{1} j_{1}}\right)=\max S(u, \alpha)=s+10$.

Let $S\left(w_{l_{0} m_{0}}, \alpha\right)=\{c, c+1\}$. Now we add the edge $u w_{l_{0} m_{0}}$ to the graph $\widehat{K}_{3,4}^{\prime}$ and we color it with color $c+2$. Clearly, we obtained an edge-coloring of the graph $\widehat{K}_{3,4}$ with colors $1, \ldots, t^{\prime}\left(t^{\prime} \geq t\right)$. Let $\beta$ be this edge-coloring. Note that for each vertex $v \in V\left(\widehat{K}_{3,4}\right) \backslash\{u\}$, $S(v, \beta)$ is an interval of integers, and $S(u, \beta)=[s, s+10] \cup\{c+2\}$ is a multiset in general.

Similarly as in the proof of the case 1 of Theorem 17 it can be shown that $i_{0} \neq i_{1}$ and $j_{0} \neq j_{1}$. Clearly, the edges $v_{i_{0}} v_{j_{0}}$ and $v_{i_{1}} v_{j_{1}}$ are independent in $K_{3,4}$. By the symmetry of the graph $\widehat{K}_{3,4}$, we may assume that $\left(i_{0}, j_{0}\right)=(1,4)$ and $\left(i_{1}, j_{1}\right)=(3,7)$.

Consider the edge $v_{1} w_{14}$. Clearly, either $\beta\left(v_{1} w_{14}\right)=s+1$ or $\beta\left(v_{1} w_{14}\right)=s+2$. If $\beta\left(v_{1} w_{14}\right)=s+1$, then the colors of all edges along the cycle $C=w_{14} v_{1} w_{17} v_{7} w_{37} v_{3} w_{34} v_{4} w_{14}$ are known. This implies that $\beta\left(u w_{17}\right)=\beta\left(u w_{34}\right)=s+5$. Hence, the added color $c+2$ is $s+5$, but this is a contradiction, since $S\left(w_{17}, \beta\right)=S\left(w_{34}, \beta\right)=[s+4, s+6]$ and in both cases $s+5$ is a middle color of the sets $S\left(w_{17}, \beta\right)$ and $S\left(w_{34}, \beta\right)$.

Now assume that $\beta\left(v_{1} w_{14}\right)=s+2$. In this case the colors of all edges along the cycle $C=w_{14} v_{1} w_{17} v_{7} w_{37} v_{3} w_{34} v_{4} w_{14}$ are also known. By the symmetry of the graph $\widehat{K}_{3,4}$, we 
may assume that $\beta\left(v_{1} w_{15}\right)=s+3$ and $\beta\left(v_{1} w_{16}\right)=s+4$. Since $\beta\left(v_{7} w_{27}\right)=s+8$, we have $\min S\left(v_{2}, \beta\right) \geq s+3$. On the other hand, since $\beta\left(v_{4} w_{24}\right)=s+2$, we have $\min S\left(v_{2}, \beta\right) \leq s+4$. We consider two cases.

Case 1: $\min S\left(v_{2}, \beta\right)=s+3$.

In this case $\beta\left(v_{2} w_{26}\right) \leq s+5$ and thus $\beta\left(u w_{36}\right)=s+9$. This implies that $\beta\left(v_{3} w_{36}\right)=s+7$ and $\beta\left(v_{6} w_{36}\right)=s+8$. Since $\beta\left(v_{1} w_{16}\right)=s+4$, we have $\beta\left(v_{6} w_{16}\right)=s+6$ and $\beta\left(v_{6} w_{26}\right)=$ $s+7$. Also, since $\beta\left(v_{2} w_{26}\right) \leq s+5$, we have $\beta\left(u w_{26}\right)=s+6$. On the other hand, $\beta\left(u w_{17}\right)=s+6$, but this is a contradiction, since $S\left(w_{17}, \beta\right)=S\left(w_{26}, \beta\right)=[s+5, s+7]$ and in both cases $s+6$ is a middle color of the sets $S\left(w_{17}, \beta\right)$ and $S\left(w_{26}, \beta\right)$.

Case 2: $\min S\left(v_{2}, \beta\right)=s+4$.

In this case $\beta\left(u w_{24}\right)=s+3$ and $\beta\left(v_{2} w_{24}\right)=s+4$. This implies that $\beta\left(v_{2} w_{25}\right) \geq s+5$ and thus $\beta\left(u w_{15}\right)=s+1$. Since $\beta\left(v_{1} w_{15}\right)=s+3$, we have $\beta\left(v_{5} w_{15}\right)=s+2$. Also, since $\beta\left(v_{3} w_{35}\right) \geq s+6$, we have $\beta\left(v_{5} w_{35}\right)=s+4$ and $\beta\left(v_{5} w_{25}\right)=s+3$. This implies that $\beta\left(u w_{25}\right)=s+4$. On the other hand, $\beta\left(u w_{34}\right)=s+4$, but this is a contradiction, since $S\left(w_{34}, \beta\right)=S\left(w_{25}, \beta\right)=[s+3, s+5]$ and in both cases $s+4$ is a middle color of the sets $S\left(w_{34}, \beta\right)$ and $S\left(w_{25}, \beta\right)$.

\section{Interval non-edge-colorable bipartite multigraphs}

In this section we consider bipartite multigraphs, and first we show that any bipartite multigraph $G$ with at most four vertices is interval colorable.

Theorem 20 If $G$ is a connected bipartite multigraph with $|V(G)| \leq 4$, then $G \in \mathfrak{N}$.

Proof. The cases $|V(G)| \leq 3$ are trivial. Assume that $|V(G)|=4$. If the underlying graph of $G$ is a tree, then the proof is trivial, too. Now let $V(G)=\{u, v, w, z\}$ and $E(G)=E(u v) \cup E(v w) \cup E(w z) \cup E(u z)$ with $\mu(u v)=a, \mu(v w)=b, \mu(w z)=c$, $\mu(u z)=d$. Without loss of generality we may assume that $\max \{a, b, c, d\}=d$. We color edges from $E(u v)$ with colors $d+1, \ldots, d+a$, edges from $E(v w)$ with colors $d-b+1, \ldots, d$, edges from $E(w z)$ with colors $d+1, \ldots, d+c$, and edges from $E(u z)$ with colors $1, \ldots, d$. If $a<c$, then the obtained coloring is an interval $(d+c)$-coloring of the multigraph $G$; otherwise the obtained coloring is an interval $(d+a)$-coloring of the multigraph $G$.

Note that the bipartite multigraph $G$ with $|V(G)|=5$ and $\Delta(G)=9$ shown in Fig. 7 has no interval coloring. Now we show a more general result.

Let us define parachute multigraphs $\operatorname{Par}\left(r_{1}, \ldots, r_{n}\right)\left(r_{1} \geq \cdots \geq r_{n} \geq 1\right)$ as follows:

$$
\begin{gathered}
V\left(\operatorname{Par}\left(r_{1}, \ldots, r_{n}\right)\right)=\left\{u, w, v_{1}, \ldots, v_{n}\right\}, \\
E\left(\operatorname{Par}\left(r_{1}, \ldots, r_{n}\right)\right)=\left\{u v_{i}: \mu\left(u v_{i}\right)=r_{i}, 1 \leq i \leq n\right\} \cup\left\{v_{j} w: 1 \leq j \leq n\right\} .
\end{gathered}
$$

Clearly, $\operatorname{Par}\left(r_{1}, \ldots, r_{n}\right)$ is a connected bipartite multigraph with $\left|\operatorname{V}\left(\operatorname{Par}\left(r_{1}, \ldots, r_{n}\right)\right)\right|=$ $n+2, \Delta\left(\operatorname{Par}\left(r_{1}, \ldots, r_{n}\right)\right)=d(u)=\sum_{i=1}^{n} r_{i}$, and $d(w)=n, d\left(v_{i}\right)=r_{i}+1, i=1,2, \ldots, n$. 


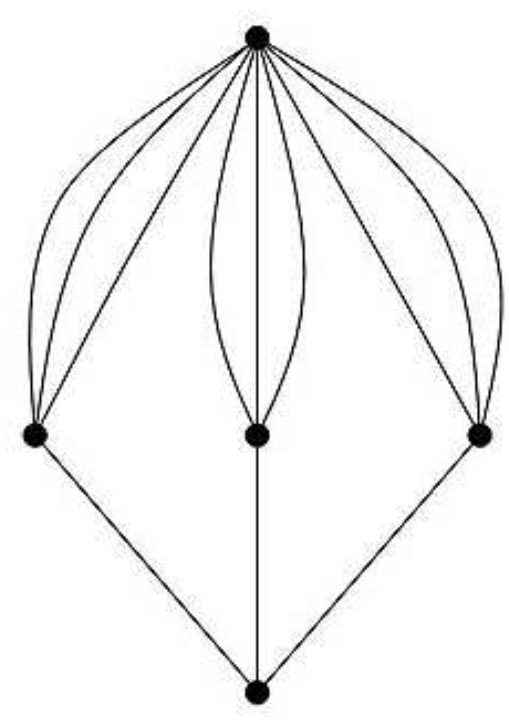

Figure 7. The bipartite multigraph $G$.

Theorem 21 If $\sum_{i=3}^{n} r_{i} \geq n+1(n \geq 3)$, then $\operatorname{Par}\left(r_{1}, \ldots, r_{n}\right) \notin \mathfrak{N}$.

Proof. Suppose, to the contrary, that the multigraph $\operatorname{Par}\left(r_{1}, \ldots, r_{n}\right)$ has an interval $t$-coloring $\alpha$ for some $t \geq \sum_{i=1}^{n} r_{i}$.

Consider the vertex $u$. Let $v_{i_{0}}$ and $v_{i_{1}}$ be two vertices adjacent to $u$ such that $\alpha\left(u v_{i_{0}}\right)=$ $\min S(u, \alpha)=s$ and $\alpha\left(u v_{i_{1}}\right)=\max S(u, \alpha)=s+\sum_{i=1}^{n} r_{i}-1$.

If $i_{0}=i_{1}$, then $\alpha\left(u v_{i_{0}}\right)=\max S(u, \alpha)=s+\sum_{i=1}^{n} r_{i}-1 \leq s+d\left(v_{i_{0}}\right)-1=s+r_{i_{0}}$ and thus $\sum_{i=1}^{n} r_{i}-1 \leq r_{i_{0}}$, which is impossible.

If $i_{0} \neq i_{1}$, then, by the construction of the multigraph $\operatorname{Par}\left(r_{1}, \ldots, r_{n}\right)$, we have

$$
\begin{gathered}
\alpha\left(v_{i_{0}} w\right) \leq s+d\left(v_{i_{0}}\right)-1=s+r_{i_{0}} \text { and thus } \\
\alpha\left(v_{i_{1}} w\right) \leq s+r_{i_{0}}+d(w)-1=s+r_{i_{0}}+n-1 .
\end{gathered}
$$

This implies that $s+\sum_{i=1}^{n} r_{i}-1=\alpha\left(u v_{i_{1}}\right)=\max S(u, \alpha) \leq s+r_{i_{0}}+n-1+d\left(v_{i_{1}}\right)-1=s+r_{i_{0}}+r_{i_{1}}+n-1$.

Hence

$$
\sum_{i=3}^{n} r_{i} \leq \sum_{i=1}^{n} r_{i}-\left(r_{i_{0}}+r_{i_{1}}\right) \leq n
$$


which is a contradiction.

By Fig. 7 and Theorem 21, we have

Corollary 22 For any positive integer $\Delta \geq 9$, there is a connected bipartite multigraph $G$ with $G \notin \mathfrak{N}$ and $\Delta(G)=\Delta$.

On the other hand, now we prove that all subcubic bipartite multigraphs have an interval coloring.

Theorem 23 If $G$ is a bipartite multigraph with $\Delta(G) \leq 3$, then $G \in \mathfrak{N}$ and $w(G) \leq 4$.

Proof. First note that if $\Delta(G) \leq 2$, then $G \in \mathfrak{N}$ and $w(G) \leq 2$.

Now suppose that $\Delta(G)=3$. For the proof, it suffices to show that $G$ has either an interval 3-coloring or an interval 4-coloring.

We show it by induction on $|E(G)|$. The statement is trivial for the case $|E(G)| \leq 4$. Assume that $|E(G)| \geq 5$, and the statement is true for all multigraphs $G^{\prime}$ with $\Delta\left(G^{\prime}\right)=3$ and $\left|E\left(G^{\prime}\right)\right|<|E(G)|$.

Let us consider a multigraph $G$. Clearly, $G$ is connected. If $G$ has no multiple edges, then the statement follows from Theorem 2. Now suppose that $G$ has multiple edges.

Let $u v \in E(G)$ and $\mu(u v) \geq 2$. We consider two cases.

Case 1: $\mu(u v)=d_{G}(v)=2$ and $d_{G}(u)=\Delta(G)=3$.

Clearly, in this case there is an edge $u w$, which is a bridge in $G$. Let us consider a multigraph $G^{\prime}=G-E(u v)$, where $E(u v)=\left\{e_{1}, e_{2}\right\}$. By induction hypothesis, $G^{\prime}$ has either an interval 3-coloring $\alpha$ or an interval 4-coloring $\alpha$.

Subcase 1.1: $\alpha(u w) \leq 2$.

We color the edge $e_{i}$ with color $\alpha(u w)+i, i=1,2$. It is not difficult to see that the obtained coloring is an interval 3-coloring or an interval 4-coloring of the multigraph $G$.

Subcase 1.2: $\alpha(u w) \geq 3$.

We color the edge $e_{i}$ with color $\alpha(u w)-i, i=1,2$. It is not difficult to see that the obtained coloring is an interval 3-coloring or an interval 4-coloring of the multigraph $G$.

Case 2: $\mu(u v)=2$ and $d_{G}(u)=d_{G}(v)=\Delta(G)=3$.

Clearly, in this case there are vertices $x, y(x \neq y)$ in $G$ such that $u x \in E(G)$ and $v y \in E(G)$. Let us consider a multigraph $G^{\prime}=(G-E(u v)-u x-v y)+x y$, where $E(u v)=\left\{e_{1}, e_{2}\right\}$. By induction hypothesis, $G^{\prime}$ has either an interval 3-coloring $\alpha$ or an interval 4-coloring $\alpha$.

Subcase 2.1: $\alpha(x y) \leq 2$.

We delete the edge $x y$ and color the edges $u x$ and $v y$ with color $\alpha(x y)$ and the edge $e_{i}$ with color $\alpha(x y)+i, i=1,2$. It is not difficult to see that the obtained coloring is an interval 3-coloring or an interval 4-coloring of the multigraph $G$.

Subcase 2.2: $\alpha(x y) \geq 3$.

We delete the edge $x y$ and color the edges $u x$ and $v y$ with color $\alpha(x y)$, and the edge $e_{i}$ with color $\alpha(x y)-i, i=1,2$. It is not difficult to see that the obtained coloring is an interval 3-coloring or an interval 4-coloring of the multigraph $G$. 


\section{Problems}

Finally, we restate the problem posed by Jensen and Toft and formulate a similar problem for multigraphs. The problems are following:

Problem 2 Is there a bipartite graph $G$ with $4 \leq \Delta(G) \leq 10$ and $G \notin \mathfrak{N}$ ?

Problem 3 Is there a bipartite multigraph $G$ with $4 \leq \Delta(G) \leq 8$ and $G \notin \mathfrak{N}$ ?

Since all bipartite graphs of order at most 14 are interval colorable [5] and the bipartite

graph $\widehat{K}_{2,2,2}$ with $\left|V\left(\widehat{K}_{2,2,2}\right)\right|=19$ is not interval colorable, we would like to suggest the following

Problem 4 Is there a bipartite graph $G$ with $15 \leq|V(G)| \leq 18$ and $G \notin \mathfrak{N}$ ?

Acknowledgement We would like to thank Rafayel R. Kamalian for his attention to this work. We also would like to thank our referees for many useful comments and suggestions which helped us to improve the presentation of the article.

\section{References}

[1] A.S. Asratian and R.R. Kamalian, Interval colorings of edges of a multigraph, Appl. Math. 5 (1987), 25-34 (in Russian).

[2] A.S. Asratian and R.R. Kamalian, Investigation on interval edge-colorings of graphs, J. Combin. Theory Ser. B 62 (1994), 34-43.

[3] A.S. Asratian, T.M.J. Denley and R. Haggkvist, Bipartite Graphs and their Applications, Cambridge University Press, Cambridge, 1998.

[4] A.S. Asratian, C.J. Casselgren, J. Vandenbussche and D.B. West, Proper path-factors and interval edge-coloring of (3,4)-biregular bigraphs, J. Graph Theory 61 (2009), 88-97.

[5] K. Giaro, Compact task scheduling on dedicated processors with no waiting periods, PhD thesis, Technical University of Gdansk, EIT faculty, Gdansk, 1999 (in Polish).

[6] K. Giaro, The complexity of consecutive $\Delta$-coloring of bipartite graphs: 4 is easy, 5 is hard, Ars Combin. 47 (1997), 287-298.

[7] K. Giaro and M. Kubale, Consecutive edge-colorings of complete and incomplete Cartesian products of graphs, Congr, Numer. 128 (1997), 143-149.

[8] K. Giaro, M. Kubale and M. Malafiejski, On the deficiency of bipartite graphs, Discrete Appl. Math. 94 (1999), 193-203.

[9] K. Giaro and M. Kubale, Compact scheduling of zero-one time operations in multistage systems, Discrete Appl. Math. 145 (2004), 95-103. 
[10] H.M. Hansen, Scheduling with minimum waiting periods, Master's Thesis, Odense University, Odense, Denmark, 1992 (in Danish).

[11] D. Hanson, C.O.M. Loten and B. Toft, On interval colorings of bi-regular bipartite graphs, Ars Combin. 50 (1998), 23-32.

[12] T.R. Jensen and B. Toft, Graph Coloring Problems, Wiley Interscience Series in Discrete Mathematics and Optimization, 1995.

[13] R.R. Kamalian, Interval colorings of complete bipartite graphs and trees, preprint, Comp. Cen. of Acad. Sci. of Armenian SSR, Erevan, 1989 (in Russian).

[14] R.R. Kamalian, Interval edge-colorings of graphs, Doctoral Thesis, Novosibirsk, 1990.

[15] R.R. Kamalian and A.N. Mirumian, Interval edge-colorings of bipartite graphs of some class, Dokl. NAN RA, 97 (1997), 3-5 (in Russian).

[16] A.V. Kostochka, unpublished manuscript, 1995.

[17] A.N. Mirumyan, personal communication, 2005.

[18] P.A. Petrosyan, Interval edge-colorings of complete graphs and $n$-dimensional cubes, Discrete Math. 310 (2010), 1580-1587.

[19] A.V. Pyatkin, Interval coloring of $(3,4)$-biregular bipartite graphs having large cubic subgraphs, J. Graph Theory 47 (2004), 122-128.

[20] S.V. Sevast'janov, Interval colorability of the edges of a bipartite graph, Metody Diskret. Analiza 50 (1990), 61-72 (in Russian).

[21] C.E. Shannon, A theorem on colouring the lines of a network, J. Math. Phys. 28 (1949), 148-151.

[22] V.G. Vizing, The chromatic class of a multigraph, Kibernetika 3 (1965), 29-39 (in Russian).

[23] D.B. West, Introduction to Graph Theory, Prentice-Hall, New Jersey, 1996.

[24] F. Yang and X. Li, Interval coloring of $(3,4)$-biregular bigraphs having two $(2,3)$ biregular bipartite subgraphs, Appl. Math. Let. 24 (2011), 1574-1577. 